\title{
Change of Scale Formulas for Wiener Integrals Related to Fourier-Feynman Transform and Convolution
}

\author{
Bong Jin Kim, ${ }^{1}$ Byoung Soo Kim, ${ }^{2}$ and Il Yoo ${ }^{3}$ \\ ${ }^{1}$ Department of Mathematics, Daejin University, Pocheon 487-711, Republic of Korea \\ ${ }^{2}$ School of Liberal Arts, Seoul National University of Science and Technology, Seoul 139-743, Republic of Korea \\ ${ }^{3}$ Department of Mathematics, Yonsei University, Wonju 220-710, Republic of Korea
}

Correspondence should be addressed to Byoung Soo Kim; mathkbs@seoultech.ac.kr

Received 18 April 2014; Accepted 19 May 2014; Published 26 June 2014

Academic Editor: Ismat Beg

Copyright (C) 2014 Bong Jin Kim et al. This is an open access article distributed under the Creative Commons Attribution License, which permits unrestricted use, distribution, and reproduction in any medium, provided the original work is properly cited.

\begin{abstract}
Cameron and Storvick discovered change of scale formulas for Wiener integrals of functionals in Banach algebra $\mathcal{S}$ on classical Wiener space. Yoo and Skoug extended these results for functionals in the Fresnel class $\mathscr{F}(B)$ and in a generalized Fresnel class $\mathscr{F}_{A 1, A 2}$ on abstract Wiener space. We express Fourier-Feynman transform and convolution product of functionals in $\mathcal{S}$ as limits of Wiener integrals. Moreover we obtain change of scale formulas for Wiener integrals related to Fourier-Feynman transform and convolution product of these functionals.
\end{abstract}

\section{Introduction}

It has long been known that Wiener measure and Wiener measurability behave badly under the change of scale transformation [1] and under translations [2]. Cameron and Storvick [3] expressed the analytic Feynman integral on classical Wiener space as a limit of Wiener integrals. In doing so, they discovered nice change of scale formulas for Wiener integrals on classical Wiener space $\left(C_{0}[0,1], m\right)[4]$. In $[5,6]$, Yoo and Skoug extended these results to an abstract Wiener space $(H, B, \nu)$. Moreover Yoo et al. [7, 8] established a change of scale formula for Wiener integrals of some unbounded functionals on (a product) abstract Wiener space. Recently Yoo et al. [9] obtained a change of scale formula for a function space integral on a generalized Wiener space $C_{a, b}[0, T]$.

On the other hand, in [10], Cameron and Storvick introduced an $L_{2}$ analytic Fourier-Feynman transform. In [11], Johnson and Skoug developed an $L_{p}$ analytic FourierFeynman transform for $1 \leq p \leq 2$ that extended the results in [10]. In [12], Huffman et al. defined a convolution product for functionals on Wiener space and, for a cylinder type functional, showed that the Fourier-Feynman transform of the convolution product is a product of Fourier-Feynman transforms. For a detailed survey of the previous work on the Fourier-Feynman transform and related topics, see [13].
In this paper, we express the Fourier-Feynman transform and convolution product of functionals in Banach algebra $\mathcal{S}$ as limits of Wiener integrals on $C_{0}[0, T]$. Moreover we obtain change of scale formulas for Wiener integrals related to Fourier-Feynman transform and convolution product of these functionals. Some preliminary results of this paper were introduced as an oral presentation in 2013 Annual Meeting of the Korean Mathematical Society [14].

Let $C_{0}[0, T]$ denote the Wiener space, that is, the space of real valued continuous functions $x$ on $[0, T]$ with $x(0)=$ 0 . Let $\mathscr{M}$ denote the class of all Wiener measurable subsets of $C_{0}[0, T]$ and let $m$ denote Wiener measure. Then $\left(C_{0}[0, T], \mathscr{M}, m\right)$ is a complete measure space and we denote the Wiener integral of a functional $F$ by

$$
\int_{C_{0}[0, T]} F(x) d m(x)
$$

A subset $E$ of $C_{0}[0, T]$ is said to be scale-invariant measurable [15] provided $\rho E$ is measurable for each $\rho>0$, and a scale-invariant measurable set $N$ is said to be scaleinvariant null provided $m(\rho N)=0$ for each $\rho>0$. A property that holds except on a scale-invariant null set is said to hold scale-invariant almost everywhere (s-a.e.). 
Let $\mathbb{C}_{+}$and $\mathbb{C}_{+}^{\sim}$ denote the sets of complex numbers with positive real part and the complex numbers with nonnegative real part, respectively. Let $F$ be a complex valued measurable functional on $C_{0}[0, T]$ such that the Wiener integral

$$
J_{F}(\lambda)=\int_{C_{0}[0, T]} F\left(\lambda^{-1 / 2} x\right) d m(x)
$$

exists as a finite number for all $\lambda>0$. If there exists a function $J_{F}^{*}(\lambda)$ analytic in $\mathbb{C}_{+}$such that $J_{F}^{*}(\lambda)=J_{F}(\lambda)$ for all $\lambda>0$, then $J_{F}^{*}(\lambda)$ is defined to be the analytic Wiener integral of $F$ over $C_{0}[0, T]$ with parameter $\lambda$, and for $\lambda \in \mathbb{C}_{+}$we write

$$
\int_{C_{0}[0, T]}^{\mathrm{anw} \mathrm{w}_{\lambda}} F(x) d m(x)=J_{F}^{*}(\lambda) .
$$

If the following limit exists for nonzero real $q$, then we call it the analytic Feynman integral of $F$ over $C_{0}[0, T]$ with parameter $q$ and we write

$$
\int_{C_{0}[0, T]}^{\mathrm{anf}_{q}} F(x) d m(x)=\lim _{\lambda \rightarrow-i q} \int_{C_{0}[0, T]}^{\mathrm{anw}_{\lambda}} F(x) d m(x),
$$

where $\lambda$ approaches $-i q$ through $\mathbb{C}_{+}$.

Now we will introduce the class of functionals that we work on in this paper. The Banach algebra $\mathcal{S}$ was introduced in [16] by Cameron and Storvick. It consists of functionals expressible in the form

$$
F(x)=\int_{L_{2}[0, T]} \exp \{i\langle v, x\rangle\} d f(v)
$$

for $s$-a.e. $x$ in $C_{0}[0, T]$, where the associated measure $f$ is a complex Borel measure on $L_{2}[0, T]$ and $\langle v, x\rangle$ denote the Paley-Wiener-Zygmund stochastic integral $\int_{0}^{T} v(t) d x(t)$.

\section{Fourier Feynman Transform and a Change of Scale Formula}

In this section we give a relationship between the Wiener integral and the Fourier-Feynman transform on $C_{0}[0, T]$ for functionals in the Banach algebra $\mathcal{S}$; that is, we express the Fourier-Feynman transform of functionals in $\delta$ as a limit of Wiener integrals on $C_{0}[0, T]$. We begin this section by introducing the definition of analytic Fourier-Feynman transform for functionals defined on $C_{0}[0, T]$. Let $1 \leq p<\infty$ and let $q$ be a nonzero real number.

Definition 1. Let $F$ be a functional on $C_{0}[0, T]$. For $\lambda \in \mathbb{C}_{+}$ and $y \in C_{0}[0, T]$, let

$$
T_{\lambda}(F)(y)=\int_{C_{0}[0, T]}^{\mathrm{anw}_{\lambda}} F(x+y) d m(x) .
$$

For $1<p<\infty$, we define the $L_{p}$ analytic Fourier-Feynman transform $T_{q}^{(p)}(F)$ of $F$ on $C_{0}[0, T]$ by the formula $\left(\lambda \in \mathbb{C}_{+}\right)$

$$
T_{q}^{(p)}(F)(y)=\underset{\lambda \rightarrow-i q}{\lim _{i} T_{\lambda}}(F)(y),
$$

whenever this limit exists; that is, for each $\rho>0$,

$$
\lim _{\lambda \rightarrow-i q} \int_{C_{0}[0, T]}\left|T_{\lambda}(F)(\rho x)-T_{q}^{(p)}(F)(\rho x)\right|^{p^{\prime}} d m(x)=0,
$$

where $1 / p+1 / p^{\prime}=1$. We define the $L_{1}$ analytic FourierFeynman transform $T_{q}^{(1)}(F)$ of $F$ by $\left(\lambda \in \mathbb{C}_{+}\right)$

$$
T_{q}^{(1)}(F)(y)=\lim _{\lambda \rightarrow-i q} T_{\lambda}(F)(y),
$$

for $s$-a.e. $y \in C_{0}[0, T]$, whenever this limit exists $[10-12,17]$.

By the definition (4) of the analytic Feynman integral and the $L_{1}$ analytic Fourier-Feynman transform (9), it is easy to see that for a nonzero real number $q$,

$$
T_{q}^{(1)}(F)(y)=\int_{C_{0}[0, T]}^{\mathrm{anf}_{q}} F(x+y) d m(x) .
$$

In particular, if $F \in \mathcal{S}$, then $F$ is analytic Feynman integrable and

$$
T_{q}^{(1)}(F)(0)=\int_{C_{0}[0, T]}^{\operatorname{anf}_{q}} F(x) d m(x) .
$$

Huffman et al. established the existence of the FourierFeynman transform on $C_{0}[0, T]$ for functionals in $\mathcal{S}$.

Theorem 2 (Theorem 3.1 of [17]). Let $F \in \mathcal{S}$ be given by (5). Then for all $\lambda \in \mathbb{C}_{+}$

$$
T_{\lambda}(F)(y)=\int_{L_{2}[0, T]} \exp \left\{i\langle v, y\rangle-\frac{1}{2 \lambda}\|v\|_{2}^{2}\right\} d f(v)
$$

for s-a.e. $y \in C_{0}[0, T]$. Moreover for all $1 \leq p<\infty$ and for all nonzero real number $q$, the Fourier-Feynman transform $T_{q}^{(p)}(F)$ exists and is given by

$$
T_{q}^{(p)}(F)(y)=\int_{L_{2}[0, T]} \exp \left\{i\langle v, y\rangle-\frac{i}{2 q}\|v\|_{2}^{2}\right\} d f(v)
$$

for s-a.e. $y \in C_{0}[0, T]$.

We next introduce an integration formula which is useful in this paper. The proof of this lemma is essentially the same as Lemma 3 of [3] and hence we will state it without proof.

Lemma 3. Let $\lambda \in \mathbb{C}_{+}$, let $\left\{\phi_{1}, \ldots, \phi_{n}\right\}$ be an orthonormal set in $L_{2}[0, T]$, and let $v \in L_{2}[0, T]$. Then

$$
\begin{gathered}
\int_{C_{0}[0, T]} \exp \left\{\frac{1-\lambda}{2} \sum_{k=1}^{n}\left\langle\phi_{k}, x\right\rangle^{2}+i\langle v, x+y\rangle\right\} d m(x) \\
=\lambda^{-n / 2} \exp \left\{\frac{\lambda-1}{2 \lambda} \sum_{k=1}^{n}\left\langle\phi_{k}, v\right\rangle^{2}-\frac{1}{2}\|v\|_{2}^{2}+i\langle v, y\rangle\right\} .
\end{gathered}
$$


Now we give a relationship between the analytic FourierFeynman transform and the Wiener integral on $C_{0}[0, T]$ for functionals in $\mathcal{S}$. In this theorem we express the FourierFeynman transform of functionals in $\delta$ as a limit of Wiener integrals.

Theorem 4. Let $F \in \mathcal{S}$ be given by (5). Let $\left\{\phi_{n}\right\}$ be a complete orthonormal set of functionals in $L_{2}[0, T]$. Let $q$ be a nonzero real number and let $\left\{\lambda_{n}\right\}$ be a sequence of complex numbers in $\mathbb{C}_{+}$such that $\lambda_{n} \rightarrow-i q$. Then we have

$$
\begin{aligned}
T_{q}^{(p)}(F)(y) & \\
=\lim _{n \rightarrow \infty} \lambda_{n}^{n / 2} \int_{C_{0}[0, T]} & \exp \left\{\frac{1-\lambda_{n}}{2} \sum_{k=1}^{n}\left\langle\phi_{k}, x\right\rangle^{2}\right\} \\
\times & F(x+y) d m(x)
\end{aligned}
$$

for s-a.e. $y \in C_{0}[0, T]$.

Proof. Let $\Gamma\left(\lambda_{n}\right)$ be the Wiener integral on the right hand side of (15). Then by (5) and the Fubini theorem,

$$
\Gamma\left(\lambda_{n}\right)=\int_{L_{2}[0, T]} K\left(v, \lambda_{n}\right) d f(v),
$$

where

$$
\begin{aligned}
K\left(v, \lambda_{n}\right)=\int_{C_{0}[0, T]} \exp \left\{\frac{1-\lambda_{n}}{2} \sum_{k=1}^{n}\left\langle\phi_{k}, x\right\rangle^{2}\right. \\
+i\langle v, x+y\rangle\} d m(x) .
\end{aligned}
$$

By Lemma 3, we evaluate the above Wiener integral to obtain

$$
\begin{aligned}
K & \left(v, \lambda_{n}\right) \\
& =\lambda_{n}^{-n / 2} \exp \left\{\frac{\lambda_{n}-1}{2 \lambda_{n}} \sum_{k=1}^{n}\left\langle\phi_{k}, v\right\rangle^{2}-\frac{1}{2}\|v\|_{2}^{2}+i\langle v, y\rangle\right\} .
\end{aligned}
$$

Now by Parseval's theorem

$$
\lim _{n \rightarrow \infty} \lambda_{n}^{n / 2} K\left(v, \lambda_{n}\right)=\exp \left\{-\frac{i}{2 q}\|v\|_{2}^{2}+i\langle v, y\rangle\right\}
$$

and so by the bounded convergence theorem

$$
\begin{aligned}
& \lim _{n \rightarrow \infty} \lambda_{n}^{n / 2} \Gamma\left(\lambda_{n}\right) \\
& \quad=\lim _{n \rightarrow \infty} \lambda_{n}^{n / 2} \int_{L_{2}[0, T]} K\left(v, \lambda_{n}\right) d f(v) \\
& \quad=\int_{L_{2}[0, T]} \exp \left\{-\frac{i}{2 q}\|v\|_{2}^{2}+i\langle v, y\rangle\right\} d f(v) .
\end{aligned}
$$

Finally by (13) in Theorem 2 , the proof is completed.
As we have seen in (10) and (11) above, if $p=1$, then the Fourier-Feynman transform $T_{q}^{(1)}(F)(0)$ is equal to the analytic Feynman integral of $F$. Hence we have the following corollary.

Corollary 5 (Theorem 2 of [3]). Let $F \in \mathcal{S}$ be given by (5). Let $\left\{\phi_{n}\right\}$ be a complete orthonormal set of functionals in $L_{2}[0, T]$. Let $q$ be a nonzero real number and let $\left\{\lambda_{n}\right\}$ be a sequence of complex numbers in $\mathbb{C}_{+}$such that $\lambda_{n} \rightarrow-i q$. Then we have

$$
\begin{aligned}
\int_{C_{0}[0, T]}^{a n f_{q}} F(x) d m(x) & \\
=\lim _{n \rightarrow \infty} \lambda_{n}^{n / 2} \int_{C_{0}[0, T]} & \exp \left\{\frac{1-\lambda_{n}}{2} \sum_{k=1}^{n}\left\langle\phi_{k}, x\right\rangle^{2}\right\} \\
& \times F(x) d m(x) .
\end{aligned}
$$

The following is a relationship between $T_{\lambda}(F)$ and the Wiener integral for functionals in $\mathcal{S}$.

Theorem 6. Let $F \in \mathcal{S}$ be given by (5). Let $\left\{\phi_{n}\right\}$ be a complete orthonormal set of functionals in $L_{2}[0, T]$. Then for each $\lambda \epsilon$ $\mathbb{C}_{+}$we have

$$
\begin{aligned}
& T_{\lambda}(F)(y) \\
&=\lim _{n \rightarrow \infty} \lambda^{n / 2} \int_{C_{0}[0, T]} \exp \left\{\frac{1-\lambda}{2} \sum_{k=1}^{n}\left\langle\phi_{k}, x\right\rangle^{2}\right\} \\
& \times F(x+y) d m(x)
\end{aligned}
$$

for s-a.e. $y \in C_{0}[0, T]$.

Proof. To prove this theorem, we modify the proof of Theorem 4 by replacing $\lambda_{n}$ by $\lambda$ whenever it occurs. Then we have

$$
\lim _{n \rightarrow \infty} \lambda^{n / 2} K(v, \lambda)=\exp \left\{-\frac{1}{2 \lambda}\|v\|_{2}^{2}+i\langle v, y\rangle\right\} .
$$

We apply the dominated convergence theorem to obtain

$$
\lim _{n \rightarrow \infty} \lambda^{n / 2} \Gamma(\lambda)=\int_{L_{2}[0, T]} \exp \left\{-\frac{1}{2 \lambda}\|v\|_{2}^{2}+i\langle v, y\rangle\right\} d f(v) .
$$

By (12) in Theorem 2, the proof is completed.

Our main result in this section, namely, a change of scale formula for Wiener integral related to Fourier-Feynman transform of functionals in $\mathcal{S}$, now follows from Theorem 6 .

Theorem 7. Let $F \in \mathcal{S}$ be given by (5). Let $\left\{\phi_{n}\right\}$ be a complete orthonormal set of functionals in $L_{2}[0, T]$. Then for each $\rho>0$

$$
\begin{aligned}
& \int_{C_{0}[0, T]} F(\rho x+y) d m(x) \\
&=\lim _{n \rightarrow \infty} \rho^{-n} \int_{C_{0}[0, T]} \exp \left\{\frac{\rho^{2}-1}{2 \rho^{2}} \sum_{k=1}^{n}\left\langle\phi_{k}, x\right\rangle^{2}\right\} \\
& \times F(x+y) d m(x)
\end{aligned}
$$

for s-a.e. $y \in C_{0}[0, T]$. 
Proof. First note that for $\lambda>0$

$$
T_{\lambda}(F)(y)=\int_{C_{0}[0, T]} F\left(\lambda^{-1 / 2} x+y\right) d m(x) .
$$

Letting $\lambda=\rho^{-2}$ in (22), we have (25) and this completes the proof.

Letting $y=0$ in (25), we have the following change of scale formula for Wiener integrals on classical Wiener space.

Corollary 8 (Theorem 2 of [4]). Let $F \in \mathcal{S}$ be given by (5). Let $\left\{\phi_{n}\right\}$ be a complete orthonormal set of functionals in $L_{2}[0, T]$. Then we have

$$
\begin{aligned}
& \int_{C_{0}[0, T]} F(\rho x) d m(x) \\
& \quad=\lim _{n \rightarrow \infty} \rho^{-n} \int_{C_{0}[0, T]} \exp \left\{\frac{\rho^{2}-1}{2 \rho^{2}} \sum_{k=1}^{n}\left\langle\phi_{k}, x\right\rangle^{2}\right\} F(x) d m(x)
\end{aligned}
$$

for each $\rho>0$.

In our next example we will explicitly compute a Wiener integral of a functional under a change of scale transformation.

Example 9. Let $\left\{\phi_{n}\right\}$ be a complete orthonormal set of functionals in $L_{2}[0, T]$. Define

$$
F(x)=\exp \left\{\alpha\left\langle\phi_{1}, x\right\rangle\right\}
$$

for $x \in C_{0}[0, T]$ and $\alpha$ is a real or complex number. We evaluate the Wiener integrals on each side of (25). The left hand side of (25) can be evaluated as follows:

$$
\begin{aligned}
L & \equiv \int_{C_{0}[0, T]} F(\rho x+y) d m(x) \\
& =\int_{C_{0}[0, T]} \exp \left\{\alpha \rho\left\langle\phi_{1}, x\right\rangle+\alpha\left\langle\phi_{1}, y\right\rangle\right\} d m(x) .
\end{aligned}
$$

By the Paley-Wiener-Zygmund theorem (see [18]), we have

$$
\begin{aligned}
L & =(2 \pi)^{-1 / 2} \exp \left\{\alpha\left\langle\phi_{1}, y\right\rangle\right\} \int_{\mathbb{R}} \exp \left\{\alpha \rho u-\frac{1}{2} u^{2}\right\} d u \\
& =\exp \left\{\frac{\alpha^{2} \rho^{2}}{2}+\alpha\left\langle\phi_{1}, y\right\rangle\right\} .
\end{aligned}
$$

Next we evaluate the Wiener integral on the right hand side of (25). Consider

$$
\begin{gathered}
R \equiv \int_{C_{0}[0, T]} \exp \left\{\frac{\rho^{2}-1}{2 \rho^{2}} \sum_{k=1}^{n}\left\langle\phi_{k}, x\right\rangle^{2}\right\} F(x+y) d m(x) \\
=\int_{C_{0}[0, T]} \exp \left\{\frac{\rho^{2}-1}{2 \rho^{2}} \sum_{k=1}^{n}\left\langle\phi_{k}, x\right\rangle^{2}\right. \\
\left.+\alpha\left\langle\phi_{1}, x\right\rangle+\alpha\left\langle\phi_{1}, y\right\rangle\right\} d m(x) .
\end{gathered}
$$

By the Paley-Wiener-Zygmund theorem again, we have

$$
\begin{aligned}
R= & (2 \pi)^{-n / 2} \exp \left\{\alpha\left\langle\phi_{1}, y\right\rangle\right\} \\
& \times \int_{\mathbb{R}^{n}} \exp \left\{\frac{\rho^{2}-1}{2 \rho^{2}} \sum_{k=1}^{n} u_{k}^{2}+\alpha u_{1}-\frac{1}{2} \sum_{k=1}^{n} u_{k}^{2}\right\} d \vec{u} \\
= & \rho^{n} \exp \left\{\frac{\alpha^{2} \rho^{2}}{2}+\alpha\left\langle\phi_{1}, y\right\rangle\right\} .
\end{aligned}
$$

Thus we have established that (25) is valid for $F(x)=$ $\exp \left\{\alpha\left\langle\phi_{1}, x\right\rangle\right\}$.

Note that in Example 9 above, $\alpha$ is a real or complex number. If $\alpha$ is pure imaginary, $F \in \mathcal{S}$ and $F$ is an example of a functional to which Theorem 7 applies. On the other hand, if the real part of $\alpha$ is not equal to 0 , then $F$ can be unbounded. Thus this example shows that the class of functionals for which (25) holds is more extensive than $\mathcal{S}$.

\section{Convolution and a Change of Scale Formula}

In this section we give a relationship between the Wiener integral and the convolution product on $C_{0}[0, T]$ for functionals in the Banach algebra $\mathcal{S}$; that is, we express the convolution product of functionals in $\mathcal{S}$ as a limit of Wiener integrals on $C_{0}[0, T]$. We start this section by introducing the definition of convolution product for functionals on $C_{0}[0, T]$.

Definition 10. Let $F$ and $G$ be functionals on $C_{0}[0, T]$. For $\lambda \epsilon$ $\mathbb{C}_{+}$and $y \in C_{0}[0, T]$, we define their convolution product $(F * G)_{\lambda}$ by

$$
(F * G)_{\lambda}(y)=\int_{C_{0}[0, T]}^{\mathrm{anw}_{\lambda}} F\left(\frac{y+x}{\sqrt{2}}\right) G\left(\frac{y-x}{\sqrt{2}}\right) d m(x)
$$

if it exists. Moreover for nonzero real number $q$, the convolution product $(F * G)_{q}$ is defined by

$$
(F * G)_{q}(y)=\int_{C_{0}[0, T]}^{\operatorname{anf}_{q}} F\left(\frac{y+x}{\sqrt{2}}\right) G\left(\frac{y-x}{\sqrt{2}}\right) d m(x)
$$

if it exists $[12,17,19,20]$.

The following is the existence theorem for the convolution product of functionals in $\mathcal{S}$ on $C_{0}[0, T]$.

Theorem 11 (Theorem 3.2 of [17]). Let $F$ and $G$ be elements of $\mathcal{S}$ with associated complex Borel measures $f$ and $g$, respectively. Then for all $\lambda \in \mathbb{C}_{+}$

$$
\begin{aligned}
&(F * G)_{\lambda}(y) \\
&=\int_{L_{2}^{2}[0, T]} \exp \left\{\frac{i}{\sqrt{2}}\langle v+w, y\rangle\right. \\
& \\
&\left.\quad-\frac{1}{4 \lambda}\|v-w\|_{2}^{2}\right\} d f(v) d g(w)
\end{aligned}
$$


for s-a.e. $y \in C_{0}[0, T]$. Moreover for all nonzero real number $q$, the convolution product $(F * G)_{q}$ exists and is given by

$$
\begin{aligned}
&(F * G)_{q}(y) \\
&=\int_{L_{2}^{2}[0, T]} \exp \left\{\frac{i}{\sqrt{2}}\langle v+w, y\rangle\right. \\
& \\
&\left.\quad-\frac{i}{4 q}\|v-w\|_{2}^{2}\right\} d f(v) d g(w)
\end{aligned}
$$

for s-a.e. $y \in C_{0}[0, T]$.

Now we give a relationship between the convolution product and the Wiener integral on $C_{0}[0, T]$ for functionals in $\mathcal{S}$. In this theorem we express the convolution product of functionals in $\mathcal{S}$ as a limit of Wiener integrals.

Theorem 12. Let $F$ and $G$ be elements of $\mathcal{S}$ with associated complex Borel measures $f$ and $g$, respectively. Let $\left\{\phi_{n}\right\}$ be a complete orthonormal set of functionals in $L_{2}[0, T]$. Let $q$ be a nonzero real number and let $\left\{\lambda_{n}\right\}$ be a sequence of complex numbers in $\mathbb{C}_{+}$such that $\lambda_{n} \rightarrow-i q$. Then we have

$$
\begin{aligned}
(F * G)_{q}(y) & \\
=\lim _{n \rightarrow \infty} \lambda_{n}^{n / 2} \int_{C_{0}[0, T]} \exp & \left\{\frac{1-\lambda_{n}}{2} \sum_{k=1}^{n}\left\langle\phi_{k}, x\right\rangle^{2}\right\} \\
& \times F\left(\frac{y+x}{\sqrt{2}}\right) G\left(\frac{y-x}{\sqrt{2}}\right) d m(x)
\end{aligned}
$$

for s-a.e. $y \in C_{0}[0, T]$.

Proof. Let $\Gamma^{*}\left(\lambda_{n}\right)$ be the Wiener integral on the right hand side of (37). Then by (5) and the Fubini theorem,

$$
\Gamma^{*}\left(\lambda_{n}\right)=\int_{L_{2}^{2}[0, T]} K^{*}\left(v, w, \lambda_{n}\right) d f(v) d g(w),
$$

where

$$
\begin{aligned}
& K^{*}\left(v, w, \lambda_{n}\right) \\
&=\int_{C_{0}[0, T]} \exp \left\{\frac{1-\lambda_{n}}{2} \sum_{k=1}^{n}\left\langle\phi_{k}, x\right\rangle^{2}\right. \\
&+\frac{i}{\sqrt{2}}\langle v-w, x\rangle \\
&\left.+\frac{i}{\sqrt{2}}\langle v+w, y\rangle\right\} d m(x) .
\end{aligned}
$$

By Lemma 3, we evaluate the above Wiener integral to obtain

$$
\begin{aligned}
& K^{*}\left(v, w, \lambda_{n}\right) \\
&=\lambda_{n}^{-n / 2} \exp \left\{\frac{\lambda_{n}-1}{4 \lambda_{n}} \sum_{k=1}^{n}\left\langle\phi_{k}, v-w\right\rangle^{2}\right. \\
& \\
&\left.\quad-\frac{1}{4}\|v-w\|_{2}^{2}+\frac{i}{\sqrt{2}}\langle v+w, y\rangle\right\} .
\end{aligned}
$$

Now by Parseval's theorem

$$
\begin{aligned}
\lim _{n \rightarrow \infty} \lambda_{n}^{n / 2} K^{*}\left(v, w, \lambda_{n}\right) \\
\quad=\exp \left\{-\frac{i}{4 q}\|v-w\|_{2}^{2}+\frac{i}{\sqrt{2}}\langle v+w, y\rangle\right\}
\end{aligned}
$$

and so by the bounded convergence theorem

$$
\begin{aligned}
\lim _{n \rightarrow \infty} \lambda_{n}^{n / 2} \Gamma^{*}\left(\lambda_{n}\right) & \\
= & \lim _{n \rightarrow \infty} \lambda_{n}^{n / 2} \int_{L_{2}^{2}[0, T]} K^{*}\left(v, w, \lambda_{n}\right) d f(v) d g(w) \\
= & \int_{L_{2}^{2}[0, T]} \exp \left\{-\frac{i}{4 q}\|v-w\|_{2}^{2}\right. \\
& \left.+\frac{i}{\sqrt{2}}\langle v+w, y\rangle\right\} d f(v) d g(w) .
\end{aligned}
$$

Finally by (36) in Theorem 11, the proof is completed.

The following is a relationship between the convolution product $(F * G)_{\lambda}$ and the Wiener integral for functionals in $\mathcal{S}$.

Theorem 13. Let $F$ and $G$ be elements of $\mathcal{S}$ with associated complex Borel measures $f$ and $g$, respectively. Let $\left\{\phi_{n}\right\}$ be a complete orthonormal set of functionals in $L_{2}[0, T]$. Then for each $\lambda \in \mathbb{C}_{+}$we have

$$
\begin{aligned}
(F * G)_{\lambda}(y) & \\
=\lim _{n \rightarrow \infty} \lambda^{n / 2} \int_{C_{0}[0, T]} \exp & \left\{\frac{1-\lambda}{2} \sum_{k=1}^{n}\left\langle\phi_{k}, x\right\rangle^{2}\right\} \\
& \times F\left(\frac{y+x}{\sqrt{2}}\right) G\left(\frac{y-x}{\sqrt{2}}\right) d m(x)
\end{aligned}
$$

for s-a.e. $y \in C_{0}[0, T]$.

Proof. To prove this theorem, we modify the proof of Theorem 12 by replacing $\lambda_{n}$ by $\lambda$ whenever it occurs. Then we have

$$
\begin{aligned}
& \lim _{n \rightarrow \infty} \lambda^{n / 2} K^{*}(v, \lambda) \\
& \quad=\exp \left\{-\frac{1}{4 \lambda}\|v-w\|_{2}^{2}+\frac{i}{\sqrt{2}}\langle v+w, y\rangle\right\} .
\end{aligned}
$$


We apply the dominated convergence theorem to obtain

$$
\begin{aligned}
& \lim _{n \rightarrow \infty} \lambda^{n / 2} \Gamma^{*}(\lambda) \\
&=\int_{L_{2}^{2}[0, T]} \exp \left\{-\frac{1}{4 \lambda}\|v-w\|_{2}^{2}\right. \\
&\left.+\frac{i}{\sqrt{2}}\langle v+w, y\rangle\right\} d f(v) d g(w) .
\end{aligned}
$$

By (35) in Theorem 11, the proof is completed.

Our main result in this section, namely, a change of scale formula for Wiener integral related to convolution product of functionals in $\mathcal{S}$, now follows from Theorem 13 .

Theorem 14. Let $F$ and $G$ be elements of $\mathcal{S}$ with associated complex Borel measures $f$ and $g$, respectively. Let $\left\{\phi_{n}\right\}$ be a complete orthonormal set of functionals in $L_{2}[0, T]$. Then for each $\rho>0$

$$
\begin{aligned}
\int_{C_{0}[0, T]} F\left(\frac{y+\rho x}{\sqrt{2}}\right) G\left(\frac{y-\rho x}{\sqrt{2}}\right) d m(x) \\
=\lim _{n \rightarrow \infty} \rho^{-n} \int_{C_{0}[0, T]} \exp \left\{\frac{\rho^{2}-1}{2 \rho^{2}} \sum_{k=1}^{n}\left\langle\phi_{k}, x\right\rangle^{2}\right\} \\
\times F\left(\frac{y+x}{\sqrt{2}}\right) G\left(\frac{y-x}{\sqrt{2}}\right) d m(x)
\end{aligned}
$$

for s-a.e. $y \in C_{0}[0, T]$.

Proof. First note that for $\lambda>0$

$$
\begin{aligned}
(F & * G)_{\lambda}(y) \\
& =\int_{C_{0}[0, T]} F\left(\frac{y+\lambda^{-1 / 2} x}{\sqrt{2}}\right) G\left(\frac{y-\lambda^{-1 / 2} x}{\sqrt{2}}\right) d m(x) .
\end{aligned}
$$

Letting $\lambda=\rho^{-2}$ in (43), we have (46) and this completes the proof.

In our final example we will explicitly compute a Wiener integral related to convolution product under a change of scale transformation.

Example 15. Let $\left\{\phi_{n}\right\}$ be a complete orthonormal set of functionals in $L_{2}[0, T]$. Define

$$
F(x)=\exp \left\{\alpha\left\langle\phi_{1}, x\right\rangle\right\}, \quad G(x)=\exp \left\{\alpha\left\langle\phi_{2}, x\right\rangle\right\}
$$

for $x \in C_{0}[0, T]$ and $\alpha$ is a real or complex number. We evaluate the Wiener integrals on each side of (46). The left hand side of (46) can be evaluated as follows:

$$
\begin{array}{r}
L \equiv \int_{C_{0}[0, T]} F\left(\frac{y+\rho x}{\sqrt{2}}\right) G\left(\frac{y-\rho x}{\sqrt{2}}\right) d m(x) \\
=\int_{C_{0}[0, T]} \exp \left\{\frac{\alpha}{\sqrt{2}}\left\langle\phi_{1}+\phi_{2}, y\right\rangle\right. \\
\left.+\frac{\alpha \rho}{\sqrt{2}}\left\langle\phi_{1}-\phi_{2}, x\right\rangle\right\} d m(x) .
\end{array}
$$

By the Paley-Wiener-Zygmund theorem, we have

$$
\begin{aligned}
L= & (2 \pi)^{-1} \exp \left\{\frac{\alpha}{\sqrt{2}}\left\langle\phi_{1}+\phi_{2}, y\right\rangle\right\} \\
& \times \int_{\mathbb{R}^{2}} \exp \left\{\frac{\alpha \rho}{\sqrt{2}}\left(u_{1}-u_{2}\right)-\frac{1}{2}\left(u_{1}^{2}+u_{2}^{2}\right)\right\} d u_{1} d u_{2} \\
= & \exp \left\{\frac{\alpha^{2} \rho^{2}}{2}+\frac{\alpha}{\sqrt{2}}\left\langle\phi_{1}+\phi_{2}, y\right\rangle\right\} .
\end{aligned}
$$

Next we evaluate the Wiener integral on the right hand side of (46):

$$
\begin{aligned}
R \equiv \int_{C_{0}[0, T]} \exp \left\{\frac{\rho^{2}-1}{2 \rho^{2}} \sum_{k=1}^{n}\left\langle\phi_{k}, x\right\rangle^{2}\right\} \\
\times F\left(\frac{y+x}{\sqrt{2}}\right) G\left(\frac{y-x}{\sqrt{2}}\right) d m(x) \\
=\int_{C_{0}[0, T]} \exp \left\{\frac{\rho^{2}-1}{2 \rho^{2}} \sum_{k=1}^{n}\left\langle\phi_{k}, x\right\rangle^{2}\right. \\
+\frac{\alpha}{\sqrt{2}}\left\langle\phi_{1}+\phi_{2}, y\right\rangle \\
\left.+\frac{\alpha}{\sqrt{2}}\left\langle\phi_{1}-\phi_{2}, x\right\rangle\right\} d m(x) .
\end{aligned}
$$

By the Paley-Wiener-Zygmund theorem, we have

$$
\begin{aligned}
& R=(2 \pi)^{-n / 2} \exp \left\{\frac{\alpha}{\sqrt{2}}\left\langle\phi_{1}+\phi_{2}, y\right\rangle\right\} \\
& \int_{\mathbb{R}^{n}} \exp \left\{\frac{\rho^{2}-1}{2 \rho^{2}} \sum_{k=1}^{n} u_{k}^{2}+\frac{\alpha}{\sqrt{2}}\left(u_{1}-u_{2}\right)-\frac{1}{2} \sum_{k=1}^{n} u_{k}^{2}\right\} d \vec{u} \\
& =\rho^{n} \exp \left\{\frac{\alpha^{2} \rho^{2}}{2}+\frac{\alpha}{\sqrt{2}}\left\langle\phi_{1}+\phi_{2}, y\right\rangle\right\} .
\end{aligned}
$$

Thus we have established that (46) is valid for $F(x)=$ $\exp \left\{\alpha\left\langle\phi_{1}, x\right\rangle\right\}$ and $G(x)=\exp \left\{\alpha\left\langle\phi_{2}, x\right\rangle\right\}$.

Note that in Example 15 above, $\alpha$ was a real or complex number. If $\alpha$ is pure imaginary, $F$ and $G$ belong to $\mathcal{S}$, so $F$ and $G$ are examples of functionals to which Theorem 14 applies. On the other hand, if the real part of $\alpha$ is not equal to 0 , then $F$ and $G$ can be unbounded. Thus this example shows that the class of functionals for which (46) holds is more extensive than $\mathcal{S}$.

\section{Conflict of Interests}

The authors declare that there is no conflict of interests regarding the publication of this paper.

\section{Acknowledgment}

This research was supported by Basic Science Research Program through the National Research Foundation of Korea 
(NRF) funded by the Ministry of Education, Science and Technology (2010-0022563).

\section{References}

[1] R. H. Cameron and W. T. Martin, "The behavior of measure and measurability under change of scale in Wiener space," Bulletin of the American Mathematical Society, vol. 53, pp. 130-137, 1947.

[2] R. H. Cameron, "The translation pathology of Wiener space," Duke Mathematical Journal, vol. 21, pp. 623-627, 1954.

[3] R. H. Cameron and D. A. Storvick, "Relationships between the Wiener integral and the analytic Feynman integral," Supplemento ai Rendiconti del Circolo Matematico di Palermo, Serie II, no. 17, pp. 117-133, 1987.

[4] R. H. Cameron and D. A. Storvick, "Change of scale formulas for Wiener integral," Supplemento ai Rendiconti del Circolo Matematico di Palermo, Serie II, no. 17, pp. 105-115, 1987.

[5] I. Yoo and D. Skoug, "A change of scale formula for Wiener integrals on abstract Wiener spaces," International Journal of Mathematics and Mathematical Sciences, vol. 17, no. 2, pp. 239247, 1994.

[6] I. Yoo and D. Skoug, "A change of scale formula for Wiener integrals on abstract Wiener spaces. II," Journal of the Korean Mathematical Society, vol. 31, no. 1, pp. 115-129, 1994.

[7] I. Yoo, T. S. Song, and B. S. Kim, "A change of scale formula for Wiener integrals of unbounded functions. II," Communications of the Korean Mathematical Society, vol. 21, no. 1, pp. 117-133, 2006.

[8] I. Yoo, T. S. Song, B. S. Kim, and K. S. Chang, "A change of scale formula for Wiener integrals of unbounded functions," The Rocky Mountain Journal of Mathematics, vol. 34, no. 1, pp. 371-389, 2004.

[9] I. Yoo, B. J. Kim, and B. S. Kim, "A change of scale formula for a function space integrals on $C_{a, b}[0, T]$," Proceedings of the American Mathematical Society, vol. 141, pp. 2729-2739, 2013.

[10] R. H. Cameron and D. A. Storvick, "An $L_{2}$ analytic FourierFeynman transform," The Michigan Mathematical Journal, vol. 23, no. 1, pp. 1-30, 1976.

[11] G. W. Johnson and D. L. Skoug, "An $L_{p}$ analytic FourierFeynman transform," The Michigan Mathematical Journal, vol. 26, no. 1, pp. 103-127, 1979.

[12] T. Huffman, C. Park, and D. Skoug, "Analytic Fourier-Feynman transforms and convolution," Transactions of the American Mathematical Society, vol. 347, no. 2, pp. 661-673, 1995.

[13] D. Skoug and D. Storvick, "A survey of results involving transforms and convolutions in function space," The Rocky Mountain Journal of Mathematics, vol. 34, no. 3, pp. 1147-1175, 2004.

[14] B. S. Kim, B. J. Kim, and I. Yoo, "Change of scale formulas for Wiener integrals related with Fourier-Feynman transform and convolution," vol. 50, p. 53, Proceedings of the Annual Meeting of the Korean Mathematical Society, 2013.

[15] G. W. Johnson and D. L. Skoug, "Scale-invariant measurability in Wiener space," Pacific Journal of Mathematics, vol. 83, no. 1, pp. 157-176, 1979.

[16] R. H. Cameron and D. A. Storvick, "Some Banach algebras of analytic Feynman integrable functionals," in Analytic Functions Kozubnik 1979, vol. 798 of Lecture Notes in Mathematics, pp. 1867, Springer, Berlin, Germany, 1980.

[17] T. Huffman, C. Park, and D. Skoug, "Convolutions and FourierFeynman transforms of functional involving multiple integrals,"
The Michigan Mathematical Journal, vol. 43, no. 2, pp. 247-261, 1996.

[18] J. Yeh, Stochastic Processes and the Wiener Integral, Marcel Dekker, New York, NY, USA, 1973.

[19] J. Yeh, "Convolution in Fourier-Wiener transform," Pacific Journal of Mathematics, vol. 15, pp. 731-738, 1965.

[20] I. Yoo, "Convolution and the Fourier-Wiener transform on abstract Wiener space," The Rocky Mountain Journal of Mathematics, vol. 25, no. 4, pp. 1577-1587, 1995. 


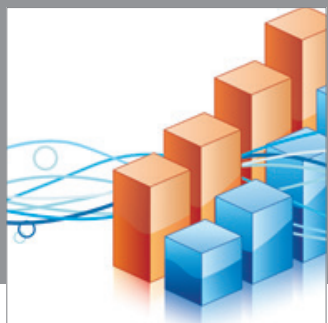

Advances in

Operations Research

mansans

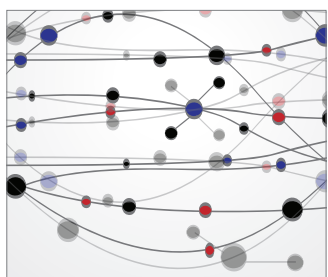

The Scientific World Journal
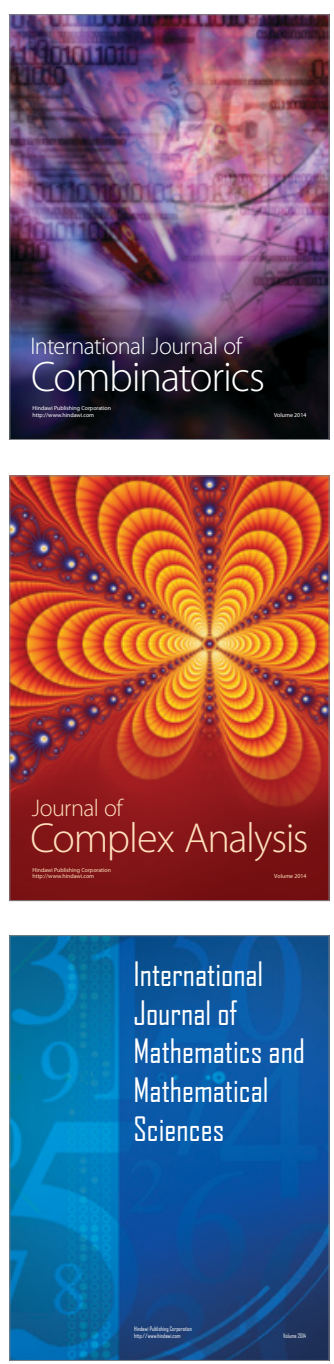
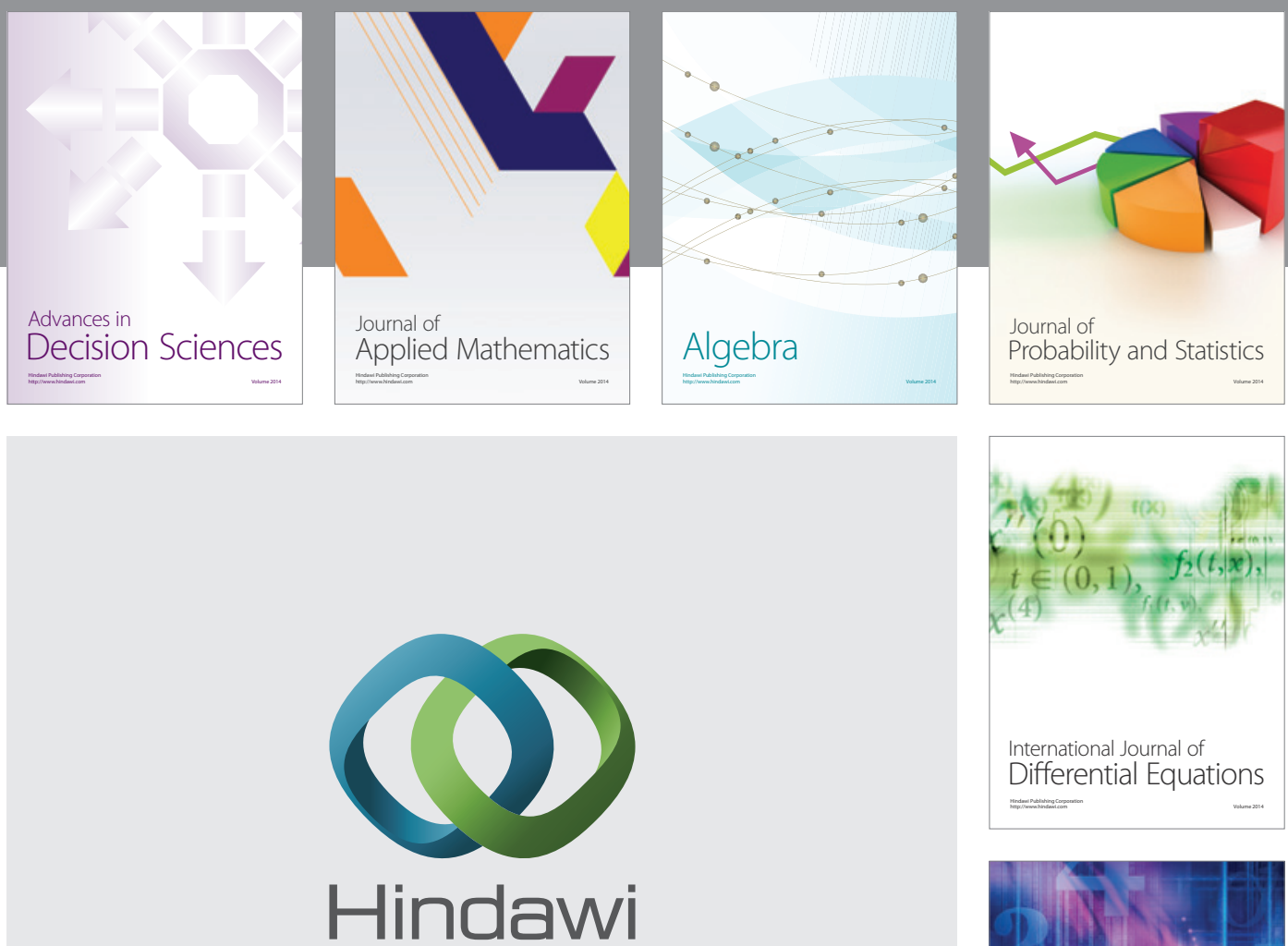

Submit your manuscripts at http://www.hindawi.com
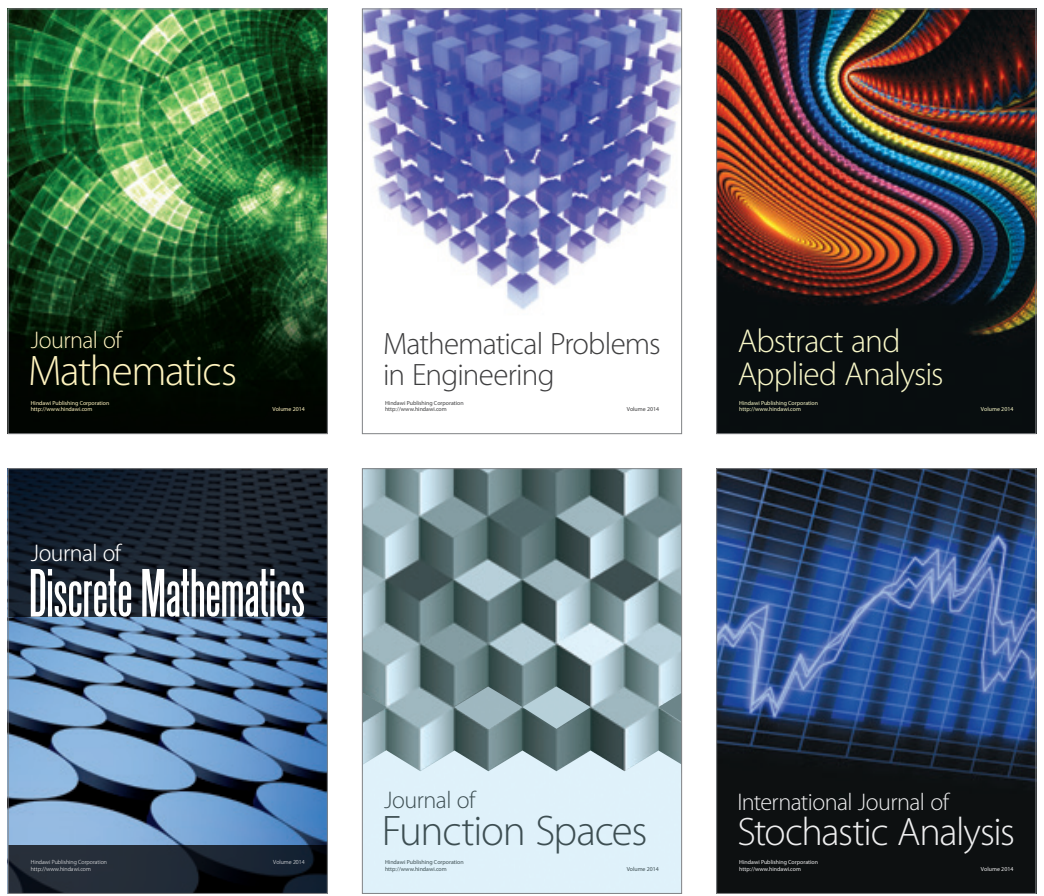

Journal of

Function Spaces

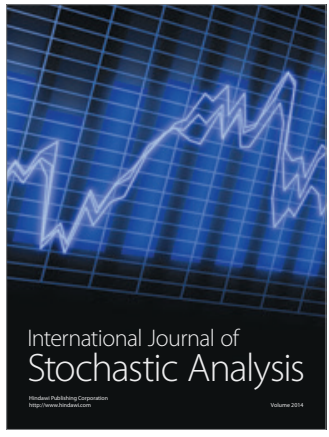

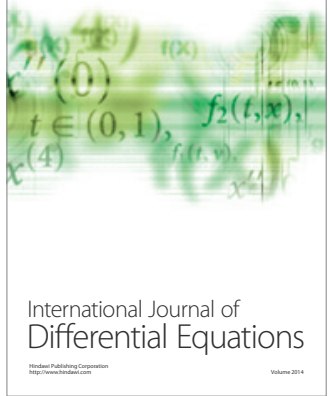
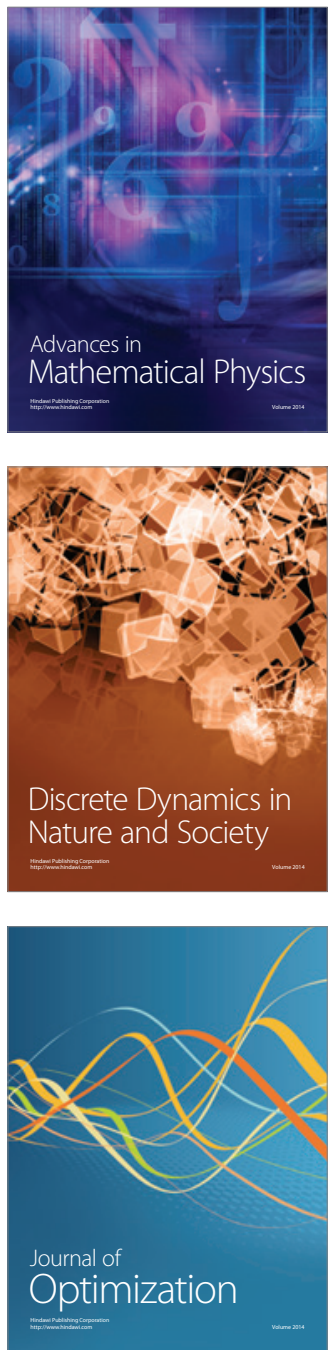